\title{
Paul Feyerabend y el problema de las entidades teóricas
}

\author{
Fernando Tula Molina
}

\section{INTRODUCGIÓN}

En lo que sigue intentaré reconstruir, y dentro de ciertos límites analizar, los principales argumentos expuestos por Paul K. Feyerabend en su artículo El problema de la existencia de las entidades teóricas, que aquí se publica traducido. ${ }^{1}$ Sin embargo, dicha reconstrucción no será tal que nos lleve a admitir, junto a Feyerabend, que el rumbo para una solución del problema considerado ha quedado definitivamente establecido. Mi propósito es, por el contrario, tomar como punto de partida la respuesta final dada por Feyerabend para luego, mirando hacia atrás intentar determinar en qué medida ha quedado establecida dicha repuesta, suponiendo que su aceptación o rechazo dependa de las tesis que fueron relacionadas por Feyerabend.

Si vale esta analogía con la lógica proposicional, mi interés no es considerar el artículo a través de las condiciones suficientes para establecer el resultado al que se arriba, sino a través de las condiciones necesarias para determinar su validez. Seguir este camino inverso, creo, permite tener presente en todo momento el objetivo perseguido y descubrir la intencionalidad de Feyerabend a lo largo de sus distintos argumentos. Teniendo en cuenta que estos últimos son muchos y de variada índole, es razonable considerar ventajosa la decisión realizada por proporcionarnos una guía para movernos entre ellos.

En primer lugar desarrollaré escuetamente el problema que se discutirá, su marco y supuestos. Luego, una vez establecida la respuesta al problema planteado, reconstruiré lo que creo es la estrategia por medio de la cual se llegó a ella. Esto, finalmente, otorgará un cierto orden en el posterior análisis de los argumentos que considero principales.

1 Mediante la sigla TE se hará referencia a la traducción del artículo de Feyerabend al que este estudio sirve de introducción. 


\section{EL PROBLEMA}

El problema de la existencia de las entidades teóricas se hace especialmente patente cuando consideramos al progreso científico como un proceso no acumulativo, esto es, cuando se destaca la existencia de ciertos momentos en el desarrollo de una disciplina científica en los que la teoría que sobreviene reemplaza a la teoría anterior redefiniendo tanto los problemas, como así también los hechos que permitirán la validación de las teorías involucradas y las pautas metodológicas que se deberán adoptar. Afirmar esto en sentido fuerte implica la inconmensurabilidad entre ambas teorías. Se deja aquí explícitamente de lado la muy importante cuestión de si la evidencia histórica nos autoriza a hacer uso del término "inconmensurabilidad" en tal sentido. La razón para ello es que sobre la base del principio holista (cf. Feyerabend, 1981 [1965a], p. 97) del significado mantenido por Feyerabend y del reconocimiento de la imposibilidad de acceder a hechos o datos observacionales puros la inconmensurabilidad se vuelve inevitable (cf. Feyerabend, 1981 [1962], p. 77) convirtiéndose en un hecho fundamental. Así, toda vez que preguntemos por las razones para adoptar una medotodología proliferacionista Feyerabend nos indicará, entre otras cosas, la necesidad de reconocer la existencia de teorías inconmensurables. Por mi parte, resta únicamente observar que el uso incondicionado que Feyerabend hace de tal término es de tal manera extremo que invita a la confusión (cf. Achinstein, 1964, p. 497).

Tales consideraciones conducen de inmediato a preguntarnos lo siguiente: en tales casos ¿qué sucede con las entidades teóricas a las que, para explicar ciertos fenómenos, remitía la teoría que fue completamente reemplazada? A fin de aclarar el sentido de esta pregunta observemos a qué llamamos entidad teórica. Tal es una entidad definida por dos notas características:

(1) No admite ser observada directamente;

(2) sabemos de su existencia y conocemos sus propiedades mediante una inferencia que realizamos dentro del contexto de una determinada teoría $T$.

Estar dentro de dicho contexto, por su parte, supone la aceptación de las observaciones realizadas en $T$ y de sus postulados (es decir, las afirmaciones admitidas sin cuestionamiento por $T$ que son utilizadas como punto de partida general para su desarrollo). El reemplazo radical de T por T' es el que nos lleva a admitir las observaciones y postulados de T' en lugar de los de $T$.

De este modo, al admitir que (a) la referencia de los términos teóricos es inferida a partir de observaciones y postulados propios de una cierta teoría y (b) existen momentos en que tales observaciones y postulados - supuestos de la inferencia - se re- 
emplazan por otros incompatibles con ellos, entonces ya no hay dificultad en ver que necesariamente la entidad descripta por el mismo término teórico antes y después del reemplazo de $T$ por $T$ ' (siempre suponemos que tal término descriptivo no fue abandonado) hará referencia a entidades diferentes (pensar, por ejemplo, en la historia del término "átomo").

Aceptado lo dicho, el sentido de nuestro problema cobra mayor claridad. ¿Qué grado de confianza es razonable que depositemos en las definiciones teóricas de los términos en cuestión? ¿Debemos considerarlas como descriptivas de entidades y propiedades de entidades realmente existentes ${ }^{2}$ en el mundo físico o como meras construcciones hipotéticas realizadas en el afán de explicar los fenómenos? El problema, entonces, es equivalente al de determinar si existen razones que nos obliguen a (o al menos nos permitan) interpretar los términos descriptivos de una teoría de modo realista. Este último es el que plantea, desarrolla e intenta resolver Feyerabend en el artículo traducido.

\section{ConteXto y supuestos}

\subsection{EL GONTEXTo}

El primer elemento a tener en cuenta dentro del contexto del problema recién planteado es que forma parte de una polémica más general que Feyerabend mantiene, principalmente, con el neopositivismo (para las consideraciones posteriores será bueno advertir que, en los argumentos empleados en dicha polémica, Feyerabend frecuentemente construye una versión por demás simplificada - y por lo mismo más débil - de sus oponentes). Siguiendo a Shapere podemos convenir en que

[...] con el propósito de demostrar como los significados de los términos se basaban en la experiencia, se hizo una distinción entre términos teóricos y términos de observación, y [que] una parte fundamental del programa del empirismo lógico consistió en el intento de mostrar cómo la primera clase de términos podía interpretarse sobre la base de la segunda (Shapere, 1985, p. 63).

2 Aquí la expresión "realmente existente" en el sentido más fuerte (del mismo modo que lo hace Feyerabend al plantear el problema), se refiere a si hay en el mundo, y con total independencia de todo contenido mental, algo que en sí mismo tenga las propiedades que nosotros le atribuímos mediante la definición del término por medio del cual lo nombramos. 
A fin de evitar ulteriores confusiones es necesario entender que los ataques que Feyerabend dirige contra tal distinción no pretenden invalidar la distinción misma, sino el uso que de ella quiso hacerse.

El segundo elemento que se debe tener en cuenta es el carácter metodológico de la discusión y de sus resultados. Es decir, el problema planteado no es tal que pueda ser resuelto haciendo referencia a cierto conjunto de hechos, sino que únicamente puede resolverse a través de la fundamentación de una determinada posición respecto de la metodología adecuada para el desarrollo de la ciencia. En tal sentido, Feyerabend argumenta:

(i) Si se intenta decidir la existencia de las entidades teóricas en base a la relación de la teoría que las define con otras teorías más generales, se está presuponiendo la validez de la interpretación realista en la teoría más general y, por ende, se toma como dado lo que se trata de solucionar;

(ii) Si se busca realizar tal decisión tanto en base a teorías como a observación, nuevamente se está suponiendo que no existe problema en interpretar de modo realista un término teórico;

Conclusión: el problema sólo puede solucionarse vía una discusión metodológi$c a$, que concierne a diferentes ideales de lo que debe ser una buena metodología científica. $^{3}$

\subsection{Los SUPuestos}

El principal supuesto del problema es que la verdad de las teorías que involucran los términos descriptivos en cuestión ya se haya establecido de algún modo, es decir, el problema justamente consiste en ver si podemos confiar en las descripciones del mundo físico que nos proporcionan aquellas teorías cuya verdad no está en discusión.

Además de este supuesto general, existen otros dos que según Feyerabend son específicos de toda presentación de este problema y que, por consiguiente, también adopta como marco de la discusión:

3 Para las razones que conducen a la adopción del realismo sui generis (no clásico) defendido por Feyerabend (cf. 1981 [1958], p. 35-6; 1981 [1960], p. 42). Para la proliferación y el realismo como principios metodológicos (cf. 1981 [1964], p. 196, 201; 1981 [1970], p. 72; 1963, p. 31). Ver además la distinción que hace Feyerabend entre diferentes concepciones realistas: la que admite tal interpretación para una teoría única (la que fue seleccionada mediante procedimientos empíricos) y la que lleva a interpretar de modo realista incluso a teorías en proliferación, para cumplir con el requisito de máxima testabilidad. Cf. Feyerabend 1981, v. 1, p. 5- . 
(1) A sua vez, el primer supuesto específico se divide en los dos siguientes:

(1a) Los objetos descriptos por los conceptos observacionales existen (es decir, no representan problema alguno);

(1b) Los conceptos teóricos son problemáticos precisamente porque no pueden ser observados.

(2) Existen las entidades teóricas, pues no todo es observable.

Estos supuestos no son otra cosa que el marco en el que el positivismo lógico ha discutido el problema. Nuevamente debemos recordar que los argumentos diseñados por Feyerabend están especificadamente dirigidos contra tal concepción.

La tesis principal afirma que

las teorías tienen significado independiente de las observaciones; las afirmaciones de observación no tienen sentido a menos que estén relacionadas con teorías. Es, por tanto, la oración de observación la que necesita interpretación y no la teoría (Feyerabend, 1965, p. 213).

El carácter de esta tesis es extremadamente polémico. Ella descansa en una concepción heterodoxa respecto de lo que debemos entender por observación, la cual surge de la llamada teoría pragmática de la observación. Creo que uno de los puntos más oscuros de la metodología propuesta por Feyerabend consiste en sus intentos de aclarar qué entiende por tal teoría. Dado que su análisis adecuado excede por completo los límites de esta introducción, notemos simplemente lo siguiente: toda situación observable es determinada por un sistema de interpretación el cual, a su vez, se conforma a partir de los principios propios de la teoría. Y la seguridad (sensación de evidencia) que sentimos frente a una situación observacional proviene de la confianza con que, en la práctica, usamos su lenguaje observacional. Ahora bien:

(1) Si toda observación se realiza necesariamente a partir de alguna teoría, no existirán otros hechos que los rescatados por sus lenguajes observacionales (se modifica, de este modo, el sentido positivista del término "hecho", es decir, se incluye en su significado un componente teórico).

(2) Si no existen hechos independientes de la teoría y no se quiere abandonar una posición realista, ella sólo podrá fundamentarse en consideraciones metodológicas (se modifica, en tal caso, el fundamento de la posición realista ortodoxa).

Tal vez pueda aclararse de algún modo esta cuestión, si tenemos en cuenta que el propósito final de la propuesta feyerabendiana consiste en combatir el dogmatismo que 
implica la aceptación de un cuerpo de enunciados observacionales único. Para ello será necesario, entre otras cosas, romper el vínculo establecido por el positivismo de modo indisoluble entre observación y significado (de modo directo con relación a los términos observacionales y mediante reglas respecto de los restantes). El modo de hacerlo consiste en considerar a la observación como una disposición a la acción (cf. Butts, 1966, p. 384). Con ello Feyerabend se aleja de los requisitos epistemológicos ortodoxos para determinar la verdad de las teorías científicas, la cual, en última instancia, descansa en una decisión práctica.

\section{Respuesta Al PROBlema: PRIMER ANÁLISIS}

Concisamente expuesto, el resultado final de la discusión y la respuesta de Feyerabend a la cuestión planteada es que existen todas las entidades descriptas por teorías con algún poder descriptivo, que provean una descripción aproximadamente correcta de los hechos en el mundo cotidiano (aclara que las teorías que sean probadas fuera del dominio de la vida cotidiana serán falsas; cf. Feyerabend, TE, p. 3o9).

A primera vista no resulta sencillo dar sentido pleno a tal afirmación. Y esto fundamentalmente por dos motivos: primero, porque supone la posesión de una imagen correcta de tal mundo a partir de la cual medir los méritos relativos de cada teoría; y segundo, porque son los mismos ejemplos que nos da Feyerabend los que nos llevan a notar que lo que llamamos mundo cotidiano en gran medida no es más que una construcción teórica. Sobre todo si tenemos en cuenta que no hay por parte de Feyerabend otras aclaraciones para determinar cuándo una teoría describe de tal modo el mundo cotidiano. Pero, más allá de ello, veamos de dónde provienen tales afirmaciones. El origen de esta tesis se encuentra en el reconocimiento de que no existe ninguna teoría científica cuyo lenguaje observacional pueda reclamar para sí el derecho a servir como parámetro objetivo con relación a los lenguajes observacionales de las restantes teorías. Todos están en un pie de igualdad. Así, creo, el modo de entender las afirmaciones de Feyerabend es como sigue.

Supongamos el clásico ejemplo conejo-pato de la psicología gestaltista. Hagamos ahora un paralelo entre percepción y teoría. Es claro que, según cada enfoque (teoría) de la figura (mundo cotidiano) que tenemos ante nosotros, veremos cosas (mundos) diferentes. Hasta aquí Feyerabend dirá que ambas teorías describen de modo aproximadamente correcto el mundo cotidiano. Sin embargo, volviendo a la situación gestáltica, no se aceptará la afirmación de quien, enfrentado a la figura especialmente diseñada, afirme ver un hipopótamo. Hay muchos puntos aquí que requieren mayor análisis, pero cada uno de ellos desemboca en innumerables sutilezas que es imposible tratar ahora. 
Lo que debe quedar de todo esto es que, del mismo modo que pueden haber descripciones diferentes pero igualmente correctas, podemos considerar la posibilidad de que existan teorías inconmensurables y, aún así, adecuadas factualmente. ${ }^{4}$ Si esto es así, surgen dos problemas acordes con la necesidad de proporcionar los siguientes criterios para saber:

(a) cuándo una teoría es adecuada;

(b) cómo decidir entre dos teorías adecuadas.

Para tratar el primer punto Feyerabend requiere eludir la necesidad de recurrir a los datos sensoriales. 5 Por ello, a pesar de su artificialidad, diseña el siguiente criterio tomando a las teorías como instrumentos predictivos:

Una teoría es un buen instrumento si la información que ella provee, junto con la información acerca de las condiciones iniciales características de un cierto dominio observacional $D o$ puede permitir a un robot sin órganos sensoriales, pero con tal información cargada en su interior, reaccionar en tal dominio exactamente del mismo modo que lo haría un ser conciente que, sin conocer tal teoría, haya sido entrenado para encontrar su camino respecto de Do y que puede responder, sobre la base de observación, muchas preguntas acerca de su entorno (Feyerabend, 1981 [1962], p. 93). 6

Con relación al segundo problema propone tres modos de elección (cf. Feyerabend, 1965 , p. 216-7):

4 Ciertas veces Feyerabend nos obliga a una lectura cuidadosa para evitar tensiones - en principio aparentes - como la siguiente:

(i) "(...) uno y el mismo conjunto de datos observacionales es compatible con teorias muy diferentes y mutuamente inconsistentes" (1981 [1962], p. 59).

(ii) “(...) teorias incomensurables pueden no poseer consecuencias comparables, sean observacionales o no. De aquí que puede no existir posibilidad alguna de encontrar una caracterización de las observaciones las cuales supone que confirman dos teorias incomensurables" (1981 [1962], p. 93).

El conflicto surge si, llevados por los múltiples usos que Feyerabend hace de las expresiones en distintos contextos, leemos "inconmensurable" en lugar de "mutuamente inconsistente" en (i), e interpretamos (ii) implicando la imposibilidad de encontrar una caracterización única de la observación que, en general, sea compatible con teorías poseedoras de postulados inconsistentes entre sí.

5 Es necesario recordar que, de acuerdo a la teoría pragmática de la observación, debemos distinguir entre las causas de la producción de una cierta oración de observación y las características del proceso de su producción por un lado y el significado de la oración producida de ésta o de otra manera, por otro.

6 Ver la acertada crítica contra tal critério realizada por Butts (1966, p. 392). 
(a) Inventar una teoría más general aún, que sirva de trasfondo para ambas teorías y que de tal modo permita una base de comparación;

(b) realizar un examen interno tendiente a establecer cuál de ellas proporciona una conexión más directa con la observación;

(c) Tomar en serio la teoría pragmática de la observación.7

Vemos que todas ellas deben leerse a partir de la prohibición de recurrir a procedimientos empíricos (observación, experimentos cruciales) a fin de adoptar una teoría antes que otra. Esto es, la elección entre lenguajes observacionales (interpretaciones de los términos descriptivos no teóricos) deberá establecerse mediante una argumentación no-observacional (metafísica) donde la exigencia de testabilidad se refiere a sus resultados, pero no a ella misma. ${ }^{\mathbf{8}}$ Queda claro que Feyerabend da una respuesta positiva a la cuestión de la interpretación realista de los términos descriptivos de la ciencia, incluyendo los teóricos. Sin embargo, de acuerdo a lo observado en la sección anterior, el sentido en el cual afirma que tales entidades existen no es el usual. Fácilmente se puede ver que esto es así considerando que el sentido fuerte en el que a diario afirmamos que algo existe en el mundo implica una cierta independencia de lo mental que, acorde al contextualismo mantenido por Feyerabend, no puede predicarse de las entidades teóricas.

Así, un uso fuerte (ordinario) del término impediría afirmar simultáneamente, y sin violar el principio (ontológico) de identidad, que:

(a) Es distinta la referencia de un mismo término descriptivo (fundamental) dentro de una cierta teoría y dentro de otra cuyos postulados sean incompatibles con la primera;

(b) ambas teorías son adecuadas fácticamente (se corresponden con el mundo).

Sin embargo, los principios metodológicos que Feyerabend nos propone no solamente permiten que esto suceda, sino que nos insta a forzar que así sea. La contradicción se diluye por el mencionado cambio en el sentido (a la par ordinario y positivista) del término "hecho". De este modo por "factualmente adecuado" Feyerabend entenderá únicamente "indistinguible experimentalmente" (cf. Feyerabend, 1981 [1962], p.59).9

${ }_{7}$ Una crítica de las tres alternativas se encuentra en Shapere (1985, p. 92-4).

8 Aquí "resultado" debe entenderse como consecuencia observacional.

9 En el mismo lugar, en páginas anteriores, Feyerabend dice que dos teorías son adecuadas fácticamente si no puede decidirse en favor de alguna de ellas sobre la base de la evidencia empírica disponible. Un punto a profundizar es en qué medida ello implica un lenguaje observacional común (o al menos compatible) a teorías mútuamente inconsis- 
Así, hablar de observación no es hablar de un registro objetivo de algo en el mundo (exterior), sino solamente de la percepción perfectamente articulada y directamente descriptible del objeto $^{10}$ (es decir, toda observación debe ser interpretada de modo realista pero en sentido psicológico). ${ }^{11}$

El punto aquí parece ser justamente el siguiente: si Feyerabend logra mostrar la inaplicabilidad de una postura realista en sentido fuerte (positivista), entonces no habrá motivo alguno que le impida proponer un nuevo matiz para el término "realismo" y argumentar en favor de una perspectiva metodológica acorde. En general, el modo de hacerlo consiste en destacar las tesis presupuestas en la concepción ortodoxa (estabilidad de los significados frente al cambio de teorías, existencia de datos sensoriales absolutamente seguros, homogeneidad de la experiencia etc.), y luego considerar contraejemplos o preceptos metodológicos que, en pro de evitar el dogmatismo científico, las invaliden.

Brevemente, ¿cómo es posible que dos conceptos empíricos, incompatibles entre sí, puedan describir adecuadamente la misma entidad? Esto es, ¿en qué sentido afirma Feyerabend que es adecuada la descripción que de un mismo hecho hacen dos teorías que, para explicarlo, aluden a entidades totalmente diferentes? La respuesta consiste en que ello es posible por el hecho de que todo lenguaje, incluso el lenguaje observacional de la ciencia, contiene elementos teóricos. Tales elementos (categorías, concepciones cosmológicas implícitas, supuestos metafísicos) dependen de la verdad de una cierta teoría que, en cuanto tal, en ningún caso puede ser garantizada de modo absoluto. Se genera con ello la posibilidad para que prima facie existan dos lenguajes observacionales que, determinados por diferentes principios categoriales, proporcionen descripciones del mundo alternativas. ${ }^{\mathbf{1 2}}$

tentes. Será necesario determinar si ello es posible teniendo en cuenta sus tesis sobre las características de todo lenguaje observacional y de toda observación. Esto, a su vez, repercutirá en la fuerza persuasiva de los argumentos en favor de una metodología proliferacionista.

10 "El que una situación observacional $s$ sea no observada por un organismo $O$ puede averiguarse (...) investigando las habilidades de $O$ para distinguir $s$ de otras situaciones diferentes; y tal investigación se hace comparando las reacciones de $O$ ante $s$ y ante las otras situaciones. Se dirá que $O$ puede distinguir entre $s$ y situaciones diferentes" (Feyerabend, 1981 [1962], p. 93). Pero, ¿en qué medida tal criterio no requeriría un observador neutro?

11 Un paralelo contemporáneo de una postura realista en sentido psicológico se encuentra en Jakendoff (1991). En este sentido, creo, Feyerabend posiblemente compartiría la siguiente afirmación de Jakendoff: “(...) perdimos nuestra inocencia: el realismo ahora involucra un elemento de elección existencial que antes no tenía” (1991, p. 4,30).

12 Tal vez lo que debería decirse es que las teorías determinadas por diferentes principios categoriales no nos proporcionan descripciones diversas del (mismo) mundo, sino que nos enfrentan con mundos diferentes. Sin embargo, Feyerabend no siempre es claro al respecto. Esto no implica una tensión entre ambas posturas (no habiendo modo de acceder al mundo independientemente de sus descripciones, sólo se trata de diferentes modos de hablar de lo mismo) pero en cierta medida dificulta la comprensión de sus afirmaciones (cf. Feyerabend, 1981 [1958], p. 31; 1981 [1960], p. 40 ss; 1981 [1962], p. 68). 


\section{La ESTRATEgia}

Consideremos ahora la estrategia seguida por Feyerabend a fin de conducirnos a la aceptación de sus conclusiones. Tal estrategia consiste en:

(1) Establecer que no hay diferencia cualitativa entre conceptos teóricos y observacionales (es decir, mostrar que todo lenguaje, incluso el observacional, contiene elementos teóricos).

(2) Construir el siguiente argumento:

(i) no hay diferencia cualitativa entre términos teóricos y observacionales;

(ii) los términos teóricos no son problemáticos, es decir, las entidades descriptas por ellos existen (suposición 1.a).

Conclusión: las entidades descriptas por lo términos teóricos también deben existir, al menos estos términos no presentan mayores problemas que los observacionales.

Se ve claramente que el problema consistirá en poder establecer la primera premisa. Para ello, Feyerabend buscará invalidar las distinciones preexistentes, principalmente positivistas, entre ambos tipos de términos.

Presentaré ahora las dos aclaraciones que, según Feyerabend, han permitido precisar tal distinción y luego, esquemáticamente, clasificaré los argumentos que nos presenta en su contra.

\subsection{Aglaragiones}

Primera aclaración: un término es observacional siempre que el valor de verdad de una proposición singular que lo contenga (sólo o acompañado de otros términos observacionales) es rápidamente determinado en base a la percepción; y un término teórico es aquel que requiere, para aceptar o rechazar una proposición singular que lo contenga (es decir, determinar su valor de verdad), además de la percepción, aceptar o rechazar teorías.

Segunda aclaración: un término es observacional si la proposición singular que lo contiene, no sólo es obtenida con rapidez y sin reflexión alguna, sino que su propia naturaleza hace que no necesite justificación posterior, es decir, son proposiciones totalmente seguras, no hipotéticas; y es teórico si la proposición singular que lo contiene requiere justificación (que sólo podrá tener lugar recurriendo a teorías). 


\subsection{Argumentos}

(1) Contra la primera aclaración: estos se dividen a su vez en:

(1.a) argumento general;

(1.b) argumento particular contra la posibilidad de justificar los términos teóricos sin hacer mención a teorías.

(2) Contra la segunda aclaración: también se subdividen en:

(2.a) argumento general;

(2.b) argumento particular contra la tesis de los datos sensoriales. Estos últimos se encuentran divididos en tres clases, destinadas a establecer tres tesis:

(2.b.1) que es posible dudar de los supuestos datos sensoriales como ser dolores, olores, sonidos etc;

(2.b.2) que los criterios que supuestamente reconocían los datos sensoriales no lograban su cometido;

(2.b.3) que aún cuando no podamos hacer aparecer una duda, la certeza psicológica es compatible con el carácter hipotético de la proposición.

A su vez, en (2.b.3) se reconocen, bajo un mismo supuesto (que no es posible eliminar los datos sensoriales si se parte del supuesto de que están ahí, independientemente de nuestras estipulaciones lingüísticas; cf. Feyerabend, TE, p. 301), diferentes argumentos acorde con los siguientes pasos de la estrategia seguida por Feyerabend:

(2.b.3.1) establecer que pasar de una proposición cierta psicológicamente a una proposición indudable epistemológicamente implica tomar una cierta decisión (como ser, aceptar que la seguridad psicológica en el momento de su producción constituye el criterio de existencia extramental del estado de cosas afirmado);

(2.b.3.2) mostrar que eliminar los datos sensoriales consiste simplemente en deshacer aquella decisión;

(2.b.3.3) proponer una decisión alternativa.

(3) Contra de la tesis de la estabilidad

Aquí Feyerabend necesitará mostrar que no existen proposiciones observacionales cuyo sentido sea independiente del cambio de teorías. Para ello sigue el siguiente camino:

(3.a) establecer cuáles son las características que debe tener un lenguaje observacional que satisfaga la tesis de la estabilidad;

(3.b) mostrar que las características son tales que no pueden ser aceptadas por la metodología científica;

(3.c) invalidar la afirmación de que el lenguaje ordinario sea estable frente al cambio de teorías. 


\section{Regonstrugción de los ARgumentos Gentrales}

Lo que intento hacer aquí es reconstruir los principales argumentos de Feyerabend dados en apoyo de los puntos señalados de su estrategia. El objetivo es facilitar la comprensión de su postura de modo que podamos reconocer dentro del conjunto qué aceptamos y quérechazamos. Me limito a una reconstrucción artificial basada en el modo en que personalmente leo tales argumentos y no necesariamente en los distintos niveles lógicos considerados por el autor. Dejo explícitamente de lado la evaluación final de cada uno. Seguiré el orden desarrollado en la sección anterior.

\subsection{CONTRA LA PRIMERA ACLARAGióN}

(1.a) Argumento general:

(i) casos de observación directa: de acuerdo al criterio propuesto, un concepto como el de "voltaje eléctrico" es un concepto observacional ya que, por ejemplo, un electricista experimentado puede averiguar el voltaje de una pila usando su dedo húmedo o su lengua sin recurrir ni hacer referencia a teoría alguna, y por lo tanto, mediante observación directa;

(ii) casos de observación indirecta: dado que las propiedades de muchas cosas debieron deducirse primero de manera complicada y luego pudieron hacerse accesibles a la observación directa (por ejemplo, la parte posterior de la luna), entonces no hay motivo para pensar que estos casos necesiten un tratamiento diferente de los de observación directa. ${ }^{\mathbf{1 3}}$

Conclusión: aplicar este criterio conduce a que todo término descriptivo de la ciencia sea observable en tal sentido y, por lo tanto, la aclaración no sirve como criterio entre conceptos teóricos y observacionales.

(1.b) argumento particular contra la posibilidad de justificar los terminos teóricos sin hacer mención a teorias:

(i) la justificación de toda teoría tiene carácter provisorio (y esto con independencia de si la proposición es o no obtenida con gran trabajo);

(ii) la aceptación de muchos de tales conceptos observacionales requiere hacer referencia a ciertas teorías (las que fueron utilizadas tanto en la medición, como en la

13 Son los preceptos mismos del método científico que conducen a procedimientos confiables y decisivos de prueba quienes proporcionan un criterio de existencia de un estado de cosas $S$. Luego, la standarización y costumbre en el uso de tal criterio lleva a que $S$ se vuelva observable en el sentido de la primera aclaración. Para el mismo argumento en Feyerabend (1981b, p. 52). 
construcción del instrumental apropiado) y, por ende, a la justificación de las teorías implicadas.

Conclusión: la primera aclaración no cumple con el fin para el cual fue formulada, es decir, distinguir entre conceptos seguros e hipotéticos.

\subsection{Contra La SEgunda AGLARAGión}

(2.a) Argumento general:

(i) el problema de la existencia de las entidades teóricas depende ahora de que podamos estar seguros de la verdad de las teorías involucradas;

(ii) existen factores que influyen en la justificación de toda proposición observacional: (a) la percepción de todo objeto depende de que se haya producido un aprendizaje correcto en el empleo de un complicado instrumento, nuestros ojos; (b) toda observación depende del estado psicológico del observador;

(iii) la justificación de tales factores sólo puede hacerse recurriendo a ciertas teorías (por ejemplo, la teoría oftalmológica o la teoría psicológica).

Conclusión: todo concepto empírico se torna teórico si aplicamos la segunda aclaración, por lo que tampoco puede utilizarse como criterio entre dichos términos.

(2.b) Argumento particular contra la tesis de los datos sensoriales:

El último punto es central. Ello justifica que sea extensamente discutido por Feyerabend en su intento de contraponer la siguiente tesis: "los datos sensoriales son, psicológicamente hablando, la consecuencia de nuestra creencia en la existencia de ciertas entidades teóricas"14 (Feyerabend, TE, p. 291).

(i) Dos ejemplos para mostrar que las sensaciones, que supuestamente constituyen los datos sensoriales, no poseen la propiedad que el positivismo les atribuye: no podemos confiar en ellas con absoluta seguridad. ${ }^{\mathbf{1 5}}$

Primer ejemplo: el aeródromo; la dificultad para determinar el umbral que divide una cierta sensación A - por exemplo, ruido - de una cierta sensación B - por exemplo, dolor (cf. Feyerabend, TE, p. 286);

Segundo ejemplo: el masoquismo; la existencia de sensaciones de dolor producidas como causa de sensaciones de placer y por lo tanto difícilmente catalogables.

14 Feyerabend (1981 [1962], p. 83) cita a TE como el lugar donde había mostrado la imposibilidad de dar cuenta del conocimiento a partir de los datos sensoriales.

15 La tesis subyacente a estos ejemplos es que las sensaciones son un fenómeno de contraste con relación a un fondo de sensaciones que, por lo general, no llegan a la conciencia. Tal sería la explicación adecuada de los ejemplos. 
(ii) Contraejemplo Tranekjaer-Rasmussen (cf. Feyerabend, TE, p. 296).

(iii) - (a) Esta premisa corresponde al primer paso de los tres que componen la estratégia de Feyerabend y tiene, a su vez, dos partes:

(1) las proposiciones sensoriales deben ser indudables;

(2) lo que se presenta de inmediato a la observación no puede ser considerado criterio de verdad de tales proposiciones, es decir, las proposiciones obtenidas con tal criterio son hipotéticas. ${ }^{\mathbf{1 6}}$

Conclusión: la certeza subjetiva no es suficiente para establecer la existencia de un determinado dato sensorial. Esto sólo puede hacerse si, además, decidimos aceptar como el único criterio para su existencia la compulsión intuitiva de hacer una determinada descripción, o la producción de tal descripción al ser interrogados, es decir, la disposición a un determinado proceder.

(b) segundo paso de la estratégia: mostrar que eliminar los datos sensoriales consiste simplemente en deshacer esa decisión: consecuencia inmediata de (a).

(c) último paso de la estratégia: propuesta de una decisión alternativa, mediante la cual deshacemos la decisión natural que no lleva a creer en la existencia de los datos sensoriales: utilizar como medio de descripción únicamente los experimentos metódicos e informes intersubjetivos que hacen posibles los resultados implicados en los experimentos, así como el conocimiento de que un lenguaje de los datos sensoriales no satisface ese criterio. Factibilidad: la realización de tal decisión será posible por el hecho empírico de que nuestra vida interior, así como la de otros hombres, muestra cierta regularidad, esto es, que existen paralelos; brevemente, que todos los instrumentos de medición a los que llamamos hombres reaccionan en su medio de acuerdo a ley. La decisión admitida no es, entonces, un ideal inalcanzable; por el contrario, "mucho más realista que las estipulaciones (sólo raramente formuladas explícitamente) en las que descansa el fundamento de la teoría de los datos sensoriales" (Feyerabend, TE, p. 301-2).

Consecuencia (adicional): tales proposiciones no pueden constituir el criterio de verificación de la ciencia ("no pueden ser públicas ni permiten planificar experimento alguno, si el problema que debe ser decidido por su intermedio está formulado en pro-

16 Y esto, a su vez, por tres razones: (a) siempre es posible que se produzcan nuevas pruebas; lo inmediato en sí mismo no excluye el error; (b) si así fuera, no se podría distinguir el criterio de existencia de un objeto, del objeto en sí mismo, es decir, no se podrá considerar satisfactoria, entonces, la respuesta de que "siento dolores" es una proposición correcta si se presentan dolores); (c) La verdad de las proposiciones sobre datos sensoriales depende de la verdad de otro conjunto de proposiciones que afirman muchas otras circunstancias, no necesariamente de naturaleza verbal (por ejemplo, que lo que vea en un determinado momento sea una pelota implica que si la arrojo contra una mesa ella debe rebotar). 
posiciones que nunca son correctamente comprendidas a menos que, de modo completamente casual, se posea la sensación correcta").

\subsection{Contra LA TESis DE LA ESTABILIDAD}

El esquema del argumento es el siguiente:

(i) características de un lenguaje que satisfaga la tesis de la estabilidad (tal lenguaje podría ser el lenguaje fisicalista de Carnap): (a) no contiene elementos teóricos; (b) es estable con relación al cambio de teorías; (c) ciertas observaciones son completamente suficientes para afirmar con absoluta seguridad la presencia de un determinado estado de cosas; (d) su aparato categorial siempre debe ser adecuado y nunca puede llegar una situación en la que nos veamos obligados a modificarlo;

(ii) un científico que busque evitar el dogmatismo debe admitir que toda proposición tiene que ser suceptible de revisión, por lo tanto, debe rechazar la tesis de la estabilidad y un lenguaje observacional como éste.

(iii) El lenguaje ordinario no satisface los requisitos de tal lenguaje. Ejemplo: hay elementos teóricos en el uso de los términos "arriba" y "abajo" dado que presupone un universo anisotrópico con una marcada dirección, es decir una teoría cosmológica. Conclusão: no existen proposiciones observacionales cuyo sentido sea independiente del cambio de teorias.

\section{ObservaGiones a PUNTOS POGo GLAROS}

En Shapere (1985) se encuentran críticas puntuales a las principales tesis de la metodología propuesta por Feyerabend (inconmensurabilidad, ${ }^{\mathbf{1}}$ carácter pragmático de la observación, ${ }^{\mathbf{1 8}}$ así como una seria acusación de relativismo). ${ }^{\mathbf{1 9}} \mathrm{El}$ análisis pormenorizado

17 “CCómo es posible criticar una teoría en función de hechos desenterrados por otra si el significado depende del contexto teórico y varía con el, especialmente sino no hay nada en común entre ambas teorías?” (Shapere, 1985, p. 93$)$.

18 "[...] debemos dar una interpretación conservadora a las declaraciones más radicales de Feyerabend, por ejemplo. que lo dado está afuera, que cada teoría posee su propia experiencia. Lo dado está, por cierto adentro todavía, y existe observación humana, experiencia, que es la misma para todas las teorías: no se trata de una observación independiente de la teoría sino de un lenguaje de observación independiente de la teoría, en contra de lo cual está Feyerabend" (Shapere, 1985, p 94).

$19 \mathrm{Al}$ tratar de asegurar la libertad de teorizar, Feyerabend ha hecho que la construcción de teorías sea demasiado libre; al despojar a las afirmaciones observacionales de todo sentido o significado independiente de las teorías, las ha privado también de todo poder de juicio sobre las teorías; deben ser interpretadas atribuyéndoles un significado, y por ende aplicando la teoría a esos significados; y estamos en libertad de interpretarlas como lo queramos: como 
de cualquiera de ellas requiere al menos un artículo específico. Lo que intento hacer aquí no es más que lo señalado en el título de esta última sección.

Primera observación: Feyerabend afirma que una teoría causal de la percepción suficientemente desarrollada puede proporcionarnos un criterio que nos asegure que la "referencia a la cosa" (Feyerabend, TE, p. 311-2) es correcta. ¿Cómo es posible que tal criterio último resida en una teoría? Es increíble que Feyerabend afirme esto cuando ha argumentado que ni aún el sentir un dolor muy fuerte puede asegurarnos que lo que sentimos es un dolor muy fuerte. Si, en el primer caso, usa el término "asegurar" en un sentido débil, me parecería más que justificados los reclamos de un teórico de los datos sensoriales que exija que él también pueda hacer uso del término en tal sentido. La dificultad sólo parece solucionarse si interpretamos la afirmación de Feyerabend diciendo únicamente que una teoría causal de la percepción puede - dentro de las limitaciones de toda teoría-explicarnos (no asegurarnos) cómo se produce la relación referencial.

Segunda observación: es necesario disponer de un criterio más claro y practicable para distinguir las teorías factualmente adecuadas de las restantes. Sin embargo, respecto del criterio para decidirnos entre teorías experimentalmente indistinguibles creo que es errónea la crítica de Butts quien reclama a toda costa un núcleo observacional común que nos permita decidir entre las teorías adecuadas empíricamente (Butts, 1966, p. 383). Si de algún modo hemos podido determinar que T1 y T2 coinciden en los hechos, mientras que no lo hace T3, tales hechos no tendrán nada más que decir con relación a las dos primeras y se entiende claramente la necesidad que Feyerabend ve de recurrir a procedimientos no observacionales.

Tercera observación: Feyerabend afirma como una de las razones que motivan la proliferación de teorías el requisito de máxima testabilidad conjugado con el hecho de que las teorías nunca están de acuerdo con la evidencia empírica disponible, debido al carácter general de toda teoría científica y al margen de error de toda observación (cf. Feyerabend, 1981 [1965b], p. 106). Tal afirmación conduce a malas interpretaciones y dificultades (dado que se encuentra en aparente conflicto con la afirmación de que dos

no pertinentes, o como apoyos a la evidencia. Al otorgar ilimitado poder de interpretación, por un lado, a aquello que permite posibilidades sin límite de interpretación, Feyerabend ha destruido la posibilidad de comparar y juzgar las teorías por referencia a la experiencia. Y al sostener que todos los significados varían con el contexto teórico, e implicar que una diferencia de significado es a fortiori una diferencia completa, una inconmensurabilidad, ha destruido la posibilidad de compararlas sobre alguna base" (Shapere, 1985, p. 105). 
teorías pueden ser indistinguibles experimentalmente (ambas acordes con la evidencia empírica disponible). Debemos entenderse por tal afirmación simplemente que toda teoría excede la evidencia empírica disponible.

Cuarta observación: el último paso de la estrategia de Feyerabend consiste en afirmar que se supone que todo lo observable existe y que no hay diferencia cualitativa entre observable y no observable, entonces también deben existir las entidades no observables. Sin embargo, hay un aspecto oculto en esta deducción, a la que considera la solución del problema-(cf. Feyerabend, TE, p. 310). Tal aspecto tiene que ver con lo que ya hemos señalado y consiste en el solapado cambio de sentido con que usa el término "existir". En un primer momento, cuando plantea el problema, nos lleva a pensar que, a lo largo del artículo averiguaremos si existen o no existen entidades que se correspondan con los términos descriptivos, no observables a simple vista, de una cierta teoría. Aquí el término es utilizado en sentido fuerte, al que llamamos positivista. En la argumentación final, por el contrario, el término "existir" ya ha experimentado el cambio referido (sentido psicológico). No obstante, queda claro, que si bien Feyerabend puede habernos convencido a lo largo de sus argumentaciones que el único uso legítimo del término es en este sentido relativizado, esto no basta para que haya proporcionado una solución al problema de la existencia de las entidades teóricas del modo como se lo planteó en un principio: ¿hay además de sillas y mesas, campos eléctricos? ¿pueden estos ser considerados como conceptos que se relacionan con objetos existentes? (cf. Feyerabend, TE, p. 278). Reconocer una limitación no es proporcionar una solución.

\section{Cita final}

Deseo concluir este estudio introductorio con una cita que puede ser esclarecedora de la idea central que guía la propuesta de Feyerabend:

[...] el examen crítico de la realidad y la existencia como un todo trae aparejado una considerable disminución de la sofisticación. Las concepciones del sentido común (sentido común tribal, sentido común homérico, el uso de nociones comunes en los lenguajes occidentales modernos) contienen ontologías sutilmente articuladas que incluyen espíritus, sueños, arco-iris, piedras, animales, festividades, justicia, destino, enfermedad, divorcios, el cielo, obligaciones, muerte, Watkins, miedo etc. Cada una de tales entidades se comportan de un modo complejo y característico que no puede reducirse a un patrón único ni explicarse en una fórmula: cada una de ellas refiere a otras entidades y procesos 
constituyendo un universo rico y variado. En tal universo la pregunta no es qué es real y qué no lo es. Tales interrogaciones ni siquiera contarán como preguntas genuinas; la pregunta es qué es lo que ocurre, en conexión con qué, y cómo (Feyerabend, 1981a, p. 1).

\author{
Fernando Tula Molina \\ Profesor de la Universidad Nacional de Quilmes, \\ investigador de CONICET, Argentina. \\ ftulamolina@gmail.com
}

\title{
REFERENGIAS BIBLIOGRÁFICAS
}

Achinstein, P. On the meaning of scientific terms. The Journal of Philosophy, 61, 17, p. 497-509, 1964 . Baumrin, B. (Ed.). Philosophy of science. The Delaware seminar. New York/London, Interscience Publishers, 1963. 2 v.

Butrs, R. E. Feyerabend and the pragmatic theory of observation. Philosophy of Science, 33, 3, p. 383-94, 1966.

Colodny, R. (Ed.). Beyond the edge of certainty. Englewood Cliffs, Prentice Hall, 1965.

Feyerabend, P. K. Das Problem der Existenz theoretischer Entitäten. In: Topitsch, E. (Ed.). Probleme der Wissenschaftstheorie. Festschrift für Victor Kraft. Wienn, Topish, 1960. p. 35-72.

How to be a good empiricist - a plea for tolerance in epistemological matters. In: BAumrin, B.

(Ed.). Philosophy of science. The Delaware seminar. New York/London, Interscience Publishers, 1963. v.2, p. 3-39.

Problems of empiricism. In: Colodny, R. (Ed.). Beyond the edge of certainty. Englewood Cliffs, Prentice Hall, 1965. p. 145-260.

Philosophical papers. Cambridge, Cambridge University Press, 1981. v. 1: Realism, rationalism \& scientific method.

Philosophical papers. Cambridge, Cambridge University Press, 1981.v. 2: Problems of empiricism. Introduction: scientific realism and philosophical realism. In: Philosophical papers.

Cambridge, Cambridge University Press, 1981a.v. 1, p. 1-33.

Historical background: some observations on the decay of philosophy of science. In:

Philosophical papers. Cambridge, Cambridge University Press, 1981b. v. 2, p. 1-33.

An attemp at a realistic interpretation of experience. In: . Philosophical papers. Cambridge,

Cambridge University Press, 1981 [1958]. v. 1, p. 17-36.

On the interpretation of scientific theories. In:

Philosophical papers. Cambridge, Cambridge

University Press, 1981 [1960].v. 1, p. 37-43.

Explanation, reduction and empiricism. In: Philosophical papers. Cambridge, Cambridge

University Press, 1981 [1962].v. 1, p. 44-96.

Realism and instrumentalism: comments on the logic of factual support. In: Philosophical papers. Cambridge, Cambridge University Press, 1981 [1964]. v. 1, p. 176-202.

On the "meaning" of scientific terms. In: . Philosophical papers. Cambridge, Cambridge

University Press, 1981 [1965a]. v. 1, p. 97-103. 
Feyerabend, P. K. Reply to criticism: comments on Smart, Sellars and Putnam. In: Philosophical papers. Cambridge, Cambridge University Press, 1981 [1965b]. v. 1, p. 104-13.

. Two models of epistemic change: Mill an Hegel. In: Philosophical papers. Cambridge, Cambridge University Press, 1981 [1970], v. 2, p. 65-79.

Hacking, I. (Ed.). Revoluciones científicas. México, FCE, 1985.

JAKENDOFF, R. The problem of reality. Nous, 25, 3, p. 411-33, 1991.

Shapere, D. Significado y cambio científico. In: HACKInG, I. (Ed.). Revoluciones científicas. México, FCE, $1985 \cdot$ p. $5^{8-113 .}$

Tорітsch, E. (Ed.). Probleme der Wissenschaftstheorie. Festschrift für Victor Kraft. Wienn, Topish, 1960. 


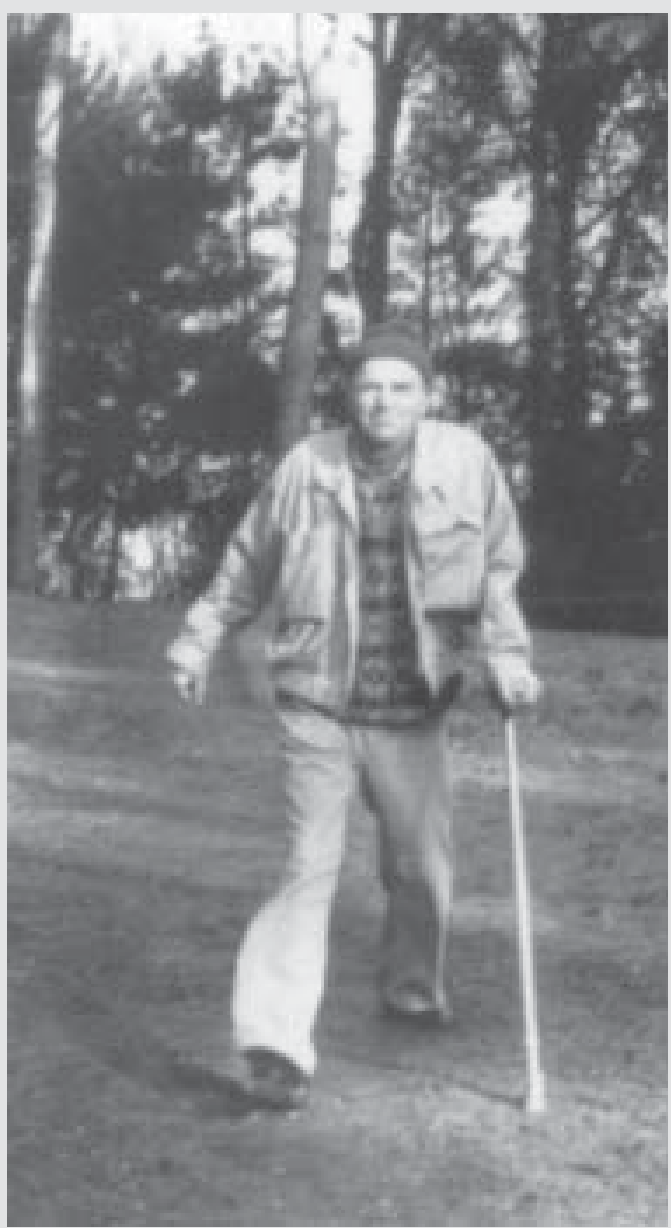

Paul Feyerabend no inverno de 1991 na Vila Ada em Roma.

Na página ao lado, retrato do final dos anos 1950, quando escreveu o artigo aqui publicado. 